\title{
El docente de Educación Superior: su práctica analizada desde las creencias del proceso de enseñanza-aprendizaje
}

\section{The higher education teacher: his practice analyzed from the beliefs about the teaching-learning process}

Enrique Navarrete Sánchez

María del Carmen Farfán García

Universidad Autónoma del

Estado de México (UAEMéx)

\author{
Ernestina Castillo De la Rosa \\ Universidad Autónoma de \\ Coahuila (UAdeC)
}

\section{Resumen}

El docente tiene un papel fundamental en el proceso de enseñanza; esta investigación se centra en el docente de Educación Superior, el cual ha tenido en la última década una serie de cambios en sus actividades, originada por las reestructuraciones de los Programas educativos y de los Planes de estudio. La transformación que puede observarse con mayor atención es la forma de concebirse la enseñanza, ya que antes el docente de este nivel educativo se contrataba para impartir docencia en su carácter de especialista de una área disciplinar, actualmente debe enseñar basado en un modelo educativo que privilegia las competencias de su disciplina, por lo tanto, el docente debe tener una serie de conocimientos que le permitan comprender su práctica educativa y determinar las estrategias didácticas que cumplan con el objetivo de su Programa, considerando la responsabilidad y el compromiso social en la formación de profesionistas que contribuyan de manera significativa al desarrollo del país y a la transformación justa de la sociedad. El objetivo del estudio fue analizar la práctica docente de un grupo de profesores de Nivel Superior de la Universidad Autónoma del Estado de México (UAEMéx.), desde las creencias del proceso enseñanza-aprendizaje. Se aplicó el Inventario de Perspectivas de Enseñanza cuyo objetivo consiste en identificar qué es lo que creen, se proponen lograr y hacen los profesores cuando enseñan. Se aplicó a una muestra de 284 docentes. Los resultados indican que existe coherencia entre lo que el profesor cree, las intenciones que tiene y las acciones del proceso de enseñanza aprendizaje.

Palabras clave: docente, educación, creencias, enseñanza, aprendizaje.

Nota del autor

Enrique Navarrete Sánchez, Facultad de Ciencias de la Conducta, Universidad Autónoma del Estado de México (UAEMéx).

María del Carmen Farfán García, Facultad de Ciencias de la Conducta, Universidad Autónoma del Estado de México (UAEMéx).

Ernestina Castillo De la Rosa, Escuela de Psicología, Universidad Autónoma de Coahuila (UAdeC). La correspondencia en relación con este artículo debe dirigirse a Enrique Navarrete Sánchez, Facultad de Ciencias de la Conducta, (UAEMéx), Filiberto Gómez S/N, (Carretera Toluca - Naucalpan Km. 1.5), Col. Guadalupe, Toluca, Estado de México, C.P. 50010.

Dirección electrónica: enavarretes@uaemex.mx Tel.: 7222720076. 


\begin{abstract}
The teacher has an essential role in the teaching process, this research is about the Higher Education teacher who has experienced a series of changes in his activities during the last decade because of the reform in Educational Programs and Study Plans. The transformation observed with more attention is the conception of teaching. Since the teacher, in this educational level, was hired to teach as a specialist of a discipline, today he/she must do it based on an educational model that favors the competencies of his discipline. Therefore, teachers must have a series of knowledge that allows them to understand his educational practice as well as to determine the didactic strategies that fulfill the objectives of the study program. Therefore, the general objective was to analyze the educational practice of a group of teachers in higher education from UAEMéx. Considering their beliefs about the teaching learning process. The Teaching Perspectives Inventory (IIPE) was administered, its objective is focused on identifying what teachers believe, what they try to achieve and what they do when they teach. The instrument was administered to 284 teachers. The findings suggest coherence between teachers' beliefs, intentions and actions in the teaching learning process.
\end{abstract}

Keywords: teacher, education, beliefs, teaching, learning.

Cuando se hace referencia al docente de Educación Superior Universitaria en México se puede referir a él por su tipo de contratación, por su categoría laboral, por la función principal que realiza en su institución, por su grado académico, etc., de allí que se deriven términos como profesor, académico, maestro, investigador; para efectos de esta investigación, se le llamará de manera indistinta, haciendo alusión a la principal actividad que realiza, la enseñanza.

Todos estos términos son para referirse a la persona que enseña, se consideran a partir de la situación actual que se vive al interior de las Instituciones de Educación Superior (IES), y que es producto de la misma dinámica de cambios sociales y estructurales de las instituciones.

Algunos de los cambios que ha sufrido la educación en términos generales en México, son las reestructuraciones y/o reformas educativas como resultado de la atención que se debe poner a los bajos resultados obtenidos en las evaluaciones educativas, tanto nacionales como internacionales. Así mismo, son consecuencia de los procesos de globalización. Producto de ello, es el cambio de los modelos educativos en los diferentes niveles, lo que conlleva un cambio de paradigma, pasando de un currículo centrado en las disciplinas y de un profesor especialista e instructor, donde la finalidad es alcanzar los objetivos, a un paradigma donde el currículo se centra en el alumno, en sus necesidades de aprendizaje, y el profesor tiene el rol de motivador y facilitador, con la finalidad de propiciar el desarrollo personal y social.

Con estos elementos y enfocados en el análisis de la práctica docente, siendo el proceso de enseñanza un rubro importante en los modelos educativos, independientemente si es el modelo 
tradicional, el modelo basado en competencias o los modelos con una estructura flexible. Lo anterior propicia una educación de calidad con un papel clave en la formación de profesionistas que contribuyan de manera significativa al desarrollo del país y a la transformación justa de la sociedad. Entendida la educación de calidad como aquella que sea: equitativa, pertinente, flexible, innovadora, diversificada y de amplia cobertura (SEP, 2015), se decidió analizar la práctica desde las creencias del docente sobre el proceso de enseñanza-aprendizaje.

Bajo esta perspectiva y a manera de contextualización, se iniciará con la importancia de la labor del docente de Educación Superior Universitaria. El profesor universitario es uno de los actores trascendentales en el proceso educativo, de tal manera que es de suma importancia determinar su concepción del proceso de enseñanza-aprendizaje como punto de partida para realizar un acercamiento al análisis, ya que para que éste se realice de manera satisfactoria se necesita del otro actor indispensable: el alumno, el cual debe querer aprender, inmerso en un proceso de construcción guiado por el docente.

Por lo anterior, el docente de Educación Superior Universitaria actualmente tiene que cumplir con ciertas características, tales como ser especialista en la disciplina que imparte, tener conocimientos de estrategias, técnicas y recursos didácticos, saber enseñar, cubrir con el programa de la unidad de aprendizaje que imparte, así como desempeñar las cuestiones administrativas de su institución referentes a su unidad de aprendizaje (juntas, evaluaciones, reporte de calificaciones, etc.). Dentro de su perfil, actualmente el docente debe mantenerse actualizado en lo que se refiere a los avances de la ciencia, en especial de su disciplina y la tecnología, mediante cursos de calidad y estudios de posgrado, es por ello que en los últimos años un gran número de docentes accede a dicho nivel de estudios.

Con relación a la función docente, es necesario considerar como lo menciona Tomás (2001, citado en Mas-Torelló \& Olmos-Rueda, 2016), "volver a pensar en la Universidad significa re conceptualizar el papel del profesorado, de los estudiantes de la enseñanza- aprendizaje, de la investigación del gobierno y de la gestión, significando este replanteamiento en la función docente 'dejar el papel de reproductor de conocimiento e ir hacia un orientador de aprendizajes...ya que también, se reorienta el aprendizaje de los estudiantes que debe permitir adquirir conocimientos pero especialmente saberlos buscar, procesar y aplicar" (p. 439).

Es por ello que, actualmente, el perfil del docente universitario requiere de una serie de características y competencias específicas, como lo menciona Mas Torelló (2011), "la responsabilidad del profesor universitario traspasa los límites del aula donde desarrolla el acto didáctico (fase interactiva); también debemos de considerar el diseño y planificación de dicha formación (fase preactiva), la evaluación de las competencias adquiridas y/o 
desarrolladas y, como no [sic], la contribución de dicho profesional a la mejora de la acción formativa desarrollada y su participación en la dinámica académico-organizativa de su institución". Al respecto, se pueden observar en la tabla 1 las seis competencias de la función docente.

\section{Tabla 1}

Seis competencias de la función docente y sus 34 unidades competenciales. (Mas Torelló, 2011)

Competencias
1. Diseñar la guía docente
de acuerdo con las
necesidades, el contexto y
el perfil profesional, todo ello
en coordinación con otros
profesionales

Función docente: unidades de competencia

1.1. Caracterizar el grupo de aprendizaje

1.2. Diagnosticar las necesidades

1.3. Formular los objetivos de acuerdo con las competencias del perfil profesional

1.4. Seleccionar y secuenciar contenidos disciplinares

1.5. Diseñar estrategias metodológicas atendiendo a la diversidad de los alumnos y la especificidad del contexto 1.6. Seleccionar y diseñar medios y recursos didácticos de acuerdo con la estrategia

1.7. Elaborar unidades didácticas de contenido

1.8. Diseñar el plan de evaluación del aprendizaje y los instrumentos necesarios

2.1. Aplicar estrategias metodológicas multivariadas, acordes con los objetivos

2.2. Utilizar diferentes medios didácticos en el proceso de enseñanza

2. Desarrollar el proceso de enseñanza-aprendizaje, propiciando oportunidades de aprendizaje tanto individuales como grupales

3.Tutorizar el proceso de aprendizaje del alumno propiciando acciones que le permitan una mayor autonomía aprendizaje

2.3. Gestionar la interacción didáctica y las relaciones con los alumnos

2.4. Establecer las condiciones óptimas y un clima social positivo para el proceso de enseñanza-aprendizaje y la comunicación

2.5. Utilizar las TIC para la combinación del trabajo presencial y no presencial del alumno

2.6. Gestionar los recursos e infraestructura aportados por la institución

2.7. Gestionar entornos virtuales de aprendizaje

3.1 Planificar acciones de tutorización, considerando los objetivos de la materia y las características de los alumnos, para optimizar el proceso de aprendizaje

3.2. Crear un clima favorable para mantener una comunicación e interacción positiva con los alumnos

3.3. Orientar, de forma individual y/o grupal, el proceso de construcción del conocimiento de los estudiantes proveyéndoles de pautas, información, recursos... para favorecer la adquisición de las competencias profesionales 3.4. Utilizar técnicas de tutorización virtual 
Tabla 1

Seis competencias de la función docente y sus 34 unidades competenciales. (Mas Torelló, 2011) (Continuación)

\begin{tabular}{|c|c|}
\hline Competencias & Función docente: unidades de competencia \\
\hline $\begin{array}{l}\text { 4. Evaluar el proceso } \\
\text { de enseñanza- } \\
\text { aprendizaje }\end{array}$ & $\begin{array}{l}\text { 4.1. Aplicar el dispositivo de evaluación de acuerdo con el plan evaluativo } \\
\text { establecido } \\
\text { 4.2. Verificar el logro de aprendizajes de los alumnos } \\
\text { 4.3. Evaluar los componentes del proceso de enseñanza-aprendizaje } \\
\text { 4.4. Promover y utilizar técnicas e instrumentos de autoevaluación discente } \\
\text { 4.5. Tomar decisiones basándose en la información obtenida } \\
\text { 4.6. Implicarse en los procesos de coevaluación } \\
\text { 4.7. Afrontar los deberes y dilemas éticos de la evaluación }\end{array}$ \\
\hline $\begin{array}{l}\text { 5. Contribuir } \\
\text { activamente a la mejora } \\
\text { de la docencia }\end{array}$ & $\begin{array}{l}\text { 5.1. Participar con otros profesionales en la concepción y elaboración de nuevos } \\
\text { instrumentos, materiales y recursos didácticos para ampliar y/o mejorar las } \\
\text { competencias profesionales } \\
\text { 5.2. Mantener relaciones con el entorno socioprofesional de forma sistemática y } \\
\text { periódica para su actualización y perfeccionamiento docente } \\
\text { 5.3. Participar activamente en acciones de innovación orientadas a la } \\
\text { optimización del proceso de aprendizaje } \\
\text { 5.4. Aplicar técnicas e instrumentos de autoevaluación docente } \\
\text { 5.5. Autodiagnosticar necesidades de formación para la mejora de la docencia }\end{array}$ \\
\hline $\begin{array}{l}\text { 6. Participar } \\
\text { activamente en la } \\
\text { dinámica académico- } \\
\text { organizativa de la } \\
\text { institución (universidad, } \\
\text { facultad, área, } \\
\text { departamento, } \\
\text { titulaciones...) }\end{array}$ & $\begin{array}{l}\text { 6.1. Participar en grupos de trabajo } \\
\text { 6.2. Participar en las comisiones multidisciplinares de docencia } \\
\text { 6.3. Promover y participar en grupos de trabajo respecto a las programaciones } \\
\text { de asignaturas pertenecientes al área de conocimiento } \\
\text { 6.4. Participar en la programación de acciones, módulos... formativos } \\
\text { 6.5. Promover la organización y participar desarrollo de jornadas académicas, } \\
\text { debates, mesas redondas... } \\
\text { 6.6. Participar en el diseño y desarrollo de los nuevos planes de estudio a partir } \\
\text { de las indicaciones, descriptores... aportados por los organismos competentes }\end{array}$ \\
\hline
\end{tabular}

Como se puede observar, para el docente actual ya no solo es suficiente ser especialista en su disciplina, sino que requiere de una serie de competencias que le permita guiar el proceso de enseñanza-aprendizaje de manera satisfactoria, destacando para ello el desarrollo de competencias psicopedagógicas; lejos quedó el profesor que dicta clases magistrales. Al respecto, Gros y Romaña (2004, p. 148) mencionan que "la profesión docente del siglo
XXI poco tendrá que ver con la imagen de un profesor subido a la tarima e impartiendo su clase frente a un grupo de alumnos".

Al conjuntar las seis competencias descritas, el denominador común es el proceso de enseñanza-aprendizaje, por lo cual es de suma importancia determinar cómo los profesores conciben la enseñanza, qué propósitos tienen al enseñar y, finalmente, cómo enseñan. 
Es por ello que en esta investigación se abordan las creencias docentes sobre el proceso de enseñanza-aprendizaje como elemento base para comprender la práctica docente del profesor universitario. Por creencias se entiende "[...] una parte de la dimensión personal, afectiva y emocional, íntimamente ligada a la propia cultura que se manifiesta en el ambiente en el que estamos y configuramos, influyendo, a su vez, en nosotros y en lo que hacemos" (Oliver, 2009). Por su parte, J. Fickel (1999, p. 2 citado en Jiménez, 2006, p. 109) menciona que las creencias son "un conjunto de potentes esquemas sobre la educación, el conocimiento, el aprendizaje y la enseñanza, a partir de las cuales los docentes toman decisiones conscientes y priorizadas". Sin duda haciendo referencia a las creencias docentes en el mismo sentido, Rojas (2014) menciona que en la literatura especializada existe una heterogeneidad de constructos teóricos para referirse a las creencias docentes: "creencias del profesor", “concepciones", “constructos personales", "significaciones", "percepciones", "teorías implícitas", "metáforas", "expectativas", etc., son algunas de las denominaciones comunes usadas para estudiar el pensamiento pedagógico del docente. Entre ellas, quizás las nociones de "creencias" y "teorías implícitas" son las más utilizadas en las investigaciones sobre pensamiento del profesor.

De esta manera, "las teorías pedagógicas son personales, reconstruidas sobre la base de conocimientos pedagógicos históricamente elaborados y transmitidos a través de la formación y en la práctica pedagógica. Por lo tanto, son una síntesis de conocimientos culturales y de experiencias personales" (De Vincenzi, 2009). Ahora bien, de acuerdo con la instrumentación de esta investigación, se utiliza el Teaching Perspectives Inventory (TPI, Pratt \& Collins, 2001) en su versión al español, titulado Inventario de Perspectivas de Enseñanza (IPE, Canto \& Rodríguez 2010), en dicho instrumento se usa el término perspectivas de enseñanza en lugar de creencias de enseñanza; por lo tanto, se hace referencia a lo que Pratt y Collins (2002) consideran como perspectivas de enseñanza: "un conjunto interrelacionado de creencias e intenciones que da dirección y justificación a nuestras acciones. De acuerdo con esta definición y con su instrumentación, la perspectiva es un término más amplio que las creencias, pues integra en su significación aspectos que se relacionan con las intenciones que se tienen y las acciones que se realizan para enseñar" (Canto, 2010).

De acuerdo con Pratt, Collins y JarvisSelinger (2001) el componente creencia se refiere a lo que el profesor cree acerca de la enseñanza; el componente acción, a lo que hacen los profesores cuando enseñan, y el componente intención, a lo que éstos pretenden lograr cuando enseñan. Cada perspectiva es una mezcla única de creencias, intenciones y acciones relacionadas con la enseñanza (Canto \& Rodríguez, 2010). 
En atención a estas consideraciones, el trabajo se centra en las creencias docentes sobre la enseñanza, cuyas características de acuerdo a Díaz, Martínez, Roa y Sanhueza (2010), con base en autores como Barry y Ammon (1996), Goodson y Numan, (2002), Kennedy (2002), Levin (2001), Muchmore (2004) Pajares (1992), Richards y Lockhart (1998) y Tillema (1998), identifican, aunque no compartidas por todos los autores, las siguientes características de las creencias:

1. Se forman tempranamente $y$ tienden $a$ auto-perpetuarse y persistir frente a la contradicción causada por el tiempo, la razón, la escolaridad o la experiencia.

2. Las personas desarrollan un sistema de creencias que alberga a todas aquellas adquiridas mediante el proceso de transmisión cultural.

3. El sistema de creencias tiene una función adaptativa que ayuda a las personas a definir y comprender el mundo y a sí mismas.

4. El conocimiento y las creencias están intrínsecamente relacionados; sin embargo, la naturaleza afectiva, evaluativa y episódica de las creencias hace que ellas se conviertan en un filtro mediante el cual se interpretan nuevos fenómenos.

5. Los procesos de pensamiento pueden muy bien ser los precursores y creadores de las creencias; no obstante, el efecto filtro de las creencias redefine, distorsiona y reestructura el procesamiento posterior de la información.
6. Las creencias epistemológicas tienen un rol fundamental en la interpretación del conocimiento y monitoreo cognitivo.

7. Las creencias se priorizan, según las conexiones o relaciones, con otras creencias u otras estructuras cognitivas y afectivas.

8. Entre más temprano se incorpora una creencia en la estructura de creencias, su modificación resulta más difícil.

9. El cambio de las creencias durante la adultez es un fenómeno relativamente raro.

10. Las creencias son instrumentales en la definición de tareas y la selección de herramientas cognitivas que sirven para interpretar, planificar y tomar decisiones con respecto a las tareas; por lo tanto, las creencias tienen un rol fundamental en la definición de un comportamiento y la organización del conocimiento y la información.

11. Las creencias influyen en la percepción, pero no son un camino confiable para acceder a la naturaleza de la realidad.

Dentro de este marco, para Crookes (2003), Hamel (2003), Harmer (1998), Moll (1993), Tillema, (1998), además de Williams y Burden (1999), citados por Díaz et al. (2010), las creencias de los docentes influyen en su actuación más que los conocimientos disciplinarios que ellos poseen. Las creencias tienden a estar limitadas culturalmente, a formarse en una época temprana de nuestra vida y a ser resistentes al cambio. Las creencias acerca de la enseñanza parecen estar bien asentadas cuando un estudiante llega a la universidad. 
Ellas están íntimamente relacionadas con lo que se cree que se sabe, pero ofrecen un filtro eficaz que discrimina, redefine, distorsiona o modifica el pensamiento y el procesamiento de la información posteriores.

De esta manera, las creencias del docente sustentan su forma de actuar en el salón de clases con respecto a su trabajo, el modo en que concibe a su alumno y al mismo proceso, el uso de estrategias y técnicas didácticas. Al determinar estas creencias se puede reflexionar y comprender el actuar del docente.

Cuando el docente de Educación Superior se integra a una IES, su fortaleza es la disciplina en que se formó y probablemente de la cual tiene experiencia. Sin embargo, tiene que aprender a enseñar, teniendo como referente principal a algún maestro que en su proceso de formación le dejó huella. Al respecto, Richards (1999), Roberts (2002) y Tsui (2003) señalan que estudiar las creencias de los docentes implica explorar el lado oculto de la enseñanza. Esto se sustenta en dos procesos socio-cognitivos. Uno de ellos está relacionado con cómo los docentes aprenden a enseñar. El otro proceso implica el tema epistemológico, relacionado con cómo los docentes saben lo que deben hacer para hacer lo que hacen. El primer punto examina lo que se conoce como el aprendizaje del docente, mientras que el segundo se relaciona con el conocimiento docente. Estos dos procesos están interrelacionados y se comunican entre sí. De hecho se podría discutir que es muy difícil conceptualizar cómo aprenden los docentes sin alguna noción de lo que están aprendiendo (Díaz et al., 2010).
Del mismo modo, Rojas (2014) mencionan que las creencias docentes acerca de la enseñanza comienzan a gestarse a partir de las propias experiencias escolares de los futuros maestros. Visto así, el objetivo de este trabajo fue analizar la práctica docente de un grupo de profesores de Nivel Superior de la UAEMéx, desde las creencias del proceso enseñanza-aprendizaje.

\section{Método}

\section{Muestra}

La presente investigación se realizó con docentes de la UAEMéx. Mediante un muestreo no probabilístico de tipo intencional, se conformó una muestra de 284 docentes de Nivel Superior; el criterio de inclusión fue ser docente activo de cualquier organismo académico de dicha universidad.

\section{Instrumento}

Se aplicó el Inventario de Perspectivas de Enseñanza (IPE), versión en español modificada del Teaching Perspectives Inventory de Pratt y Collins (2001), cuyo objetivo se centra en identificar qué es lo que creen, que se proponen lograr y que hacen los profesores cuando enseñan.

El IPE está conformado por 45 reactivos con una categoría de respuestas que va de 1 al 5, subdividido en cinco perspectivas: Transmisión, Aprendizaje, Desarrollo, Acompañamiento y Reforma social, con nueve reactivos cada una. A su vez, cada perspectiva puede ser observada desde tres dimensiones, que representan las Creencias, las Intenciones y las Acciones que los profesores realizan cuando enseñan. Cada dimensión se responde de acuerdo con una escala de cinco puntos. Las Creencias, con una categoría 
de respuesta que va de 1 (Completamente en desacuerdo) a 5 (Completamente de acuerdo). Las dimensiones Propósitos y Acciones con una categoría de cinco puntos que va de 1 (Nunca) a 5 (Siempre). El instrumento presenta una confiabilidad total de .90 .

\section{Análisis de datos}

Ya aplicado el instrumento se llevó a cabo el vaciado de datos en el programa SPSS, dividiéndolos en diversas variables a continuación descritas:

I. Formación y profesionalización docente (primeros reactivos 1 a 6): número de sujeto, universidad a la que pertenece, función principal, tiempo de docencia que dedica a la semana, grado académico, área de especialización, asignaturas que imparte, años dedicado a la docencia; siendo cada uno de ellos una variable distinta.

II. Creencia (reactivos 1 a 15).

III. Propósitos (reactivos 16 a 30).

IV. Acciones (reactivos 31 a 45).

\section{Resultados}

La tabla 2 indica que $74.6 \%$ de la muestra se dedica a la docencia como función principal en la institución; $14.4 \%$ se dedica a investigación; y $10.2 \%$ se dedica a actividades de gestión.

En relación con el tiempo dedicado a la docencia, se tiene que $44.7 \%$ de la muestra dedica de 11 a 20 horas a la docencia; $28.2 \%$, de 1 a 10 horas; $21.1 \%$, dedica de 21 a 30 horas; y $6.0 \%$ imparte docencia de 31 a 40 horas.

Tabla 2

Función principal dentro de su escuela

\begin{tabular}{lcc}
\hline Función & Frecuencia & Porcentaje \\
\hline Docencia & 212 & 74.6 \\
Gestión & 29 & 10.2 \\
Investigación & 43 & 15.1 \\
Total & 284 & 100.0 \\
\hline
\end{tabular}

Tabla 3

Aproximadamente cuánto tiempo de su trabajo dedica a la docencia a la semana

\begin{tabular}{lcc}
\hline Horas & Frecuencia & Porcentaje \\
\hline 1 a 10 Horas & 80 & 28.2 \\
11 a 20 Horas & 127 & 44.7 \\
21 a 30 Horas & 60 & 21.1 \\
31 a 40 Horas & 17 & 6.0 \\
Total & 284 & 100.0 \\
\hline
\end{tabular}


En cuanto a los grados académicos, se tiene que $38.7 \%$ tiene una escolaridad de Licenciatura, $34.5 \%$ tiene grado de Maestría, 16.5, estudios de especialización y el $10.2 \%$ cuenta con grado de doctor.

En relación con los años dedicados a la docencia, los docentes reportan que de 9 a 16 años $36.3 \%$; $32.7 \%$ de 1 a 8 años; $25 \%$ de 17 a 24 años; y $6 \% 25$ años o más.

Los puntajes obtenidos se pueden observar en la tabla 6: las creencias tienen una media de 3.86 con una desviación típica de .388 , los propósitos tiene una media de 3.98 y una desviación típica de .229 y las acciones tiene una media de 3.85 y una desviación típica de .311 .

Tabla 4

Grado académico

\begin{tabular}{lcc}
\hline Grado académico & Frecuencia & Porcentaje \\
\hline Licenciatura & 110 & 38.7 \\
Especialización & 47 & 16.5 \\
Maestría & 98 & 34.5 \\
Doctorado & 29 & 10.2 \\
Total & 284 & 100.0 \\
\hline
\end{tabular}

Tabla 5

Años dedicados a la docencia

\begin{tabular}{lcc}
\hline Años & Frecuencia & Porcentaje \\
\hline 1 A 8 años & 93 & 32.7 \\
9 A 16 años & 103 & 36.3 \\
17 A 24 años & 71 & 25.0 \\
25 años o más & 17 & 6.0 \\
Total & 284 & 100.0 \\
\hline
\end{tabular}

Tabla 6

Medias y Desviación estándar de las dimensiones docentes

\begin{tabular}{lcl}
\hline Dimensiones & $M$ & $D E$ \\
\hline Creencias & 3.86 & .388 \\
Propósitos & 3.98 & .229 \\
Acciones & 3.85 & .311 \\
\hline
\end{tabular}




\section{Discusión}

Como bien se sabe, la realidad actual de un profesor universitario es que su función primordial y la cual le da entrada a alguna institución universitaria es la docencia, posteriormente, de acuerdo con su tipo de contratación, puede realizar investigación y/o alguna otra actividad y mejorar así su condición laboral. Asimismo, 44.7\% de la muestra dedica de 11 a 20 horas a la docencia, es decir, la docencia ocupa la mayor parte del tiempo de los profesores. Quienes dan prioridad al proceso de enseñanza y aprendizaje de los conocimientos y/o habilidades en una disciplina: $38.7 \%$ tiene una escolaridad de Licenciatura y $34.5 \%$ tiene grado de Maestría, acorde con las actuales políticas educativas de calidad, referidas al profesorado, donde se busca que su perfil le permita contar con los elementos suficientes en su formación para conducir el proceso antes referido. El 36.3\% tiene entre 9 y 16 años dedicados a la docencia, este porcentaje muestra que los profesores tienen experiencia en la conducción de dicho proceso.

Ahora bien, con respecto a las dimensiones que mide el instrumento se obtuvo que las Creencias tienen una media de 3.86 con una desviación típica de .388 , los Propósitos tienen una media de 3.98 y una desviación típica de .229 y las Acciones tienen una media de $3.85 \mathrm{y}$ una desviación típica de .311 . De acuerdo con la literatura revisada, existe coherencia entre lo que el profesor cree, las intensiones que tiene y las acciones del proceso de enseñanza aprendizaje, es así que para Pratt (2002), el término "perspectivas de enseñanza", en lugar de creencias acerca de la enseñanza, se define como "un conjunto interrelacionado de creencias e intenciones que dan dirección y justificación a nuestras acciones". Asimismo, con base en los resultados, se considera que el docente, como lo menciona Mas Torelló (2011), puede desarrollar el proceso de enseñanza-aprendizaje, al propiciar oportunidades de aprendizaje tanto individual como grupal.

A partir de los resultados, se concluye que la principal actividad del profesor universitario es la docencia, con base en ello y para comprender la práctica educativa, es necesario determinar las creencias que el profesor tiene sobre la enseñanza. Los resultados obtenidos coinciden con las características de las creencias, donde se determina que son instrumentales en la definición de tareas y la selección de herramientas cognitivas con las cuales interpretar, planificar y tomar decisiones con respecto a las tareas. Por lo tanto, las creencias tienen un rol fundamental en la definición de un comportamiento y la organización del conocimiento y la información.

De acuerdo con esto, aunado al modelo educativo, debe haber coincidencia con las creencias y formas de enseñar del profesor, por consiguiente, no basta con que se produzcan cambios curriculares, o se implementen nuevos modelos de enseñanza, se deben determinar las creencias del profesor o perspectivas de 
enseñanza, de manera que se ajuste a los propósitos educativos. Finalmente, si el profesor universitario reflexiona sobre su práctica docente está en la línea de mejorarla como un proceso continuo.

\section{Referencias}

Canto, \& Rodríguez, E. (2010). Creencias de profesores de educación superior acerca del proceso de enseñanza y aprendizaje. Informe de investigación. UADY. Documento inédito. Recuperado de http://educacionyciencia. org/index.php/educacionyciencia/article/ viewFile/276/pdf

De Vincenzi, A. (2009). Concepciones de enseñanza y su relación con las prácticas docentes: un estudio con profesores universitarios. Educación y Educadores, 12(2), 87-101. Recuperado de http:/www. redalyc.org/pdf/834/83412219006.pdf

Díaz, C., Martínez, P., Roa, I., \& Sanhueza, G. (2010). Los docentes en la sociedad actual: sus creencias y cogniciones pedagógicas respecto al proceso didáctico. POLIS, Revista Latinoamericana, 25, 1-14. Recuperado de: https://journals.openedition.org/polis/625

Gros, B., \& Romaña, T. (2004). Ser profesor. Palabras sobre la docencia universitaria. Barcelona: Ediciones Octaedro-ICE Universitat de Barcelona.

Jiménez, A. \& Feliciano, L. (2006). Pensar el pensamiento del profesorado. Revista Española de Pedagogía, 233, 105-122.
Mas-Torelló, O. (2011). El profesor universitario: sus competencias y formación. Profesorado. Revista de Currículum y Formación de Profesorado, 15(3), 195-211. Recuperado de http://www.ugr.es/ recfpro/rev153COL1.pdf Mas- Torelló O. \& Olmos- Rueda, P. (2016). El profesor universitario en el espacio Europeo de educación superior. La autopercepción de sus competencias docentes actuales y orientaciones para su formación pedagógica. Revista Mexicana de Investigación Educativa, 21(69), 437470. Recuperado de http://www.redalyc.org/ html/140/14045395005/

Oliver, C. (2009). El valor formativo y las ataduras de las creencias en la formación del profesorado. Aquello que no se ve, pero se percibe en el aula. Revista Electrónica Interuniversitaria de Formación del Profesorado, 12(1), 63-75. Recuperado de http://www.aufop.com/aufop/uploaded_files/ articulos/1240872744.pdf

Pratt, D. D. (2002).Good teaching: one size fits all? In An Up-date on Teaching Theory, Jovita Ross-Gordon (Ed.), San Francisco: Jossey-Bass, Publishers.

Pratt, D. D., \& Collins, J. B. (2001). Teaching perspectives inventory. Recuperado el 8 de octubre de 2008 de http:// teachingperspectives.com

Pratt, D. D., Collins, J. B. \& Jarvis Selinger, S. (2001). Development and Use of the Teaching Perspectives Inventory (TPI). In 
American Educational Research Association (AERA 2001) Meeting (pp.1-9). Seattle, WA. Recuperado de https://facultycommons. macewan.ca/wp-content/uploads/TPIonline-resource.pdf.

Rojas, T. (2014). Las creencias docentes: delimitación del concepto y propuesta para la investigación. Revista Electrónica Diálogos Educativos, 14(27), 89-112. Recuperado de http:/www.dialogoseducativos.cl/revistas/ n27/rojas.pdf

Secretaría de Educación Pública (2015) ¿Qué es la Subsecretaría de Educación Superior? Recuperado de: https://www.gob.mx/ sep/articulos/que-es-la-subsecretaria-deeducacion-superior

Recepción primera versión: 06/07/2017

Entrega segunda versión: 15/12/2017

Ultimas correcciones de autor: 15/05/2018 